\title{
Hitting Time and Place of Brownian Motion with Drift
}

\author{
Chuancun Yin* and Chunwei Wang
}

School of Mathematical Sciences, Qufu Normal University, Qufu 273165, China

\begin{abstract}
We consider a $d$-dimensional Brownian motion in $\mathbb{R}^{d}$ with drift. The explicit expressions are obtained for the joint density of the hitting time and place to a sphere, when the process starts either from the inside the sphere or from the out of sphere.
\end{abstract}

Keywords: Brownian motion with drift, hitting time, hitting place, Joint density.

\section{INTRODUCTION}

Let $\{X(t), t \geq 0\}$ be a standard $d$-dimensional Brownian motion with drift $c: X(t)=B(t)+c t, \quad t \geq 0$, where $B(t)$ is the standard $d$-dimensional Brownian motion, $c \in \mathbb{R}^{d}(d \geq 2)$ is a fixed vector. Let us denote by $P_{x}^{c}(\bullet)$ the probability measure on the path space of $X$ corresponding to initial value $X(0)=x$ and drift vector $c, E_{x}^{c}(\bullet)$ the corresponding expectation operator. For simplicity, we shall write $P_{x}(\bullet)$ and $E_{x}(\bullet)$ to refer the case $c=0$. For $r>0$, consider the sphere $\partial B_{r}=\left\{x: x \in \mathbb{R}^{d},|x|=r\right\}$. The first hitting time of $X$ through $\partial B_{r}$ is defined as $T_{r}=\inf \{t>0$ :

$|X(t)|=r\}$. As usual, we take $\inf \{\varnothing\}=+\infty$. The first hitting place is $X\left(T_{r}\right)$. Because of the sample path continuity of the process, $X\left(T_{r}\right)$ lies on $\partial B_{r}$.

A Laplace-Gegenbauer transform of the first hitting time and the first hitting place to a sphere centered at the origin was found by Wendel [1]; Betz and Gzyl [2, 3] gave another proof to Wendel's exterior problem. Yin [4] extened Wendel's results to the case of Brownian motion with constant drift. The joint density of the first hitting time and the first hitting place of a sphere by Brownian motion which starts at any point inside the sphere was obtained by Hsu [5]. The aim of this paper is to obtain the joint density of the first hitting time and the first hitting place of a sphere by Brownian motion with or without drift which starts at any point in space.

The following notation can be found in [6]. Let $J_{v}$ and $N_{v}$ denote the first and second Bessel function of order $v$, respectively. Let $I_{v}$ and $K_{v}$ denote the first and

*Address correspondence to this author at the School of Mathematical Sciences, Qufu Normal University, Qufu 273165, China;

E-mail: ccyin@mail.qfnu.edu.cn second Bessel function of purely imaginary argument, respectively. Let $C_{m}^{v}$ be the Gegenbauer polynomial of degree $m$ and order $v$, which is defined via its generating function: $\left(1-2 \beta t+\beta^{2}\right)^{-v}=\sum_{n=0}^{\infty} C_{n}^{v}(t) \beta^{n}$. It is customary to take $C_{0}^{0}=1, C_{0}^{v}=1, C_{m}^{0}=2 T_{m} / m$, here $T_{m}$ is the $m$ th Tchebycheff polynomial: $T_{m}(\cos \theta)=\cos m \theta$. Set $h=(d-2) / 2$.

We use $\left\{q_{m, n}, n \geq 1\right\}$ to denote the positive zeros of $J_{m+h}$ in the ascending order.

\section{LEMMAS}

In this section we give several lemmas for latter use.

Lemma 2.1. ([6]) Let $\sigma(d y)$ be the $d-1$ dimensional volume measure on $\partial B_{r}(d \geq 2)$, then

$$
\begin{aligned}
& \int_{\partial B_{r}} C_{m}^{h}(\cos \theta) C_{k}^{h}(\cos \theta) \sigma(d y) \\
& =\left\{\begin{array}{cc}
\frac{2 \pi^{\frac{d}{2}} r^{d-1} h}{(m+h) \Gamma\left(\frac{d}{2}\right)} C_{m}^{h}(1), & m=k, d \geq 3, \\
\frac{2 \pi r}{m} C_{m}^{0}(1), & m=k \neq 0, d=2, \\
2 \pi r, & m=k=0, d=2, \\
0, & m \neq k, d \geq 2,
\end{array}\right.
\end{aligned}
$$

where $\theta=\angle x 0 y, x \in \mathbb{R}^{d}$.

Lemma 2.2. ([5]) For $|x|<r, \alpha>0$ and $d \geq 2$, then

$$
-2 \sum_{n=0}^{\infty} \frac{q_{m, n} J_{m+h}\left(\frac{|x|}{r} q_{m, n}\right)}{\left(2 r^{2} \alpha+q_{m, n}^{2}\right) J_{m+h}^{\prime}\left(q_{m, n}\right)}=\frac{I_{m+h}(\sqrt{2 \alpha}|x|)}{I_{m+h}(\sqrt{2 \alpha} r)},
$$

where $m \geq 0$ is an integer.

Lemma 2.3. For $|x|>r, \quad \alpha>0$ and $d \geq 2$, then 
$\int_{0}^{\infty} \frac{\lambda\left(J_{m+h}(\lambda|x|) N_{m+h}(\lambda r)-J_{m+h}(\lambda r) N_{m+h}(\lambda|x|)\right)}{\left(\lambda^{2}+2 \alpha\right)\left(J_{m+h}^{2}(\lambda r)+N_{m+h}^{2}(\lambda r)\right)} d \lambda=-\frac{\pi}{2} \frac{K_{m+h}(\sqrt{2 \alpha}|x|)}{K_{m+h}(\sqrt{2 \alpha} r)}$, where $m \geq 0$ is an integer.

Proof. Using the recurrence formulas (see [6]):

$\frac{d}{d x}\left(x^{v} K_{v}\right)=-x^{v} K_{v-1}, \frac{d}{d x}\left(x^{-v} K_{v}\right)=-x^{-v} K_{v+1}, \frac{d}{d x}\left(x^{v} Z_{v}\right)=-x^{v} Z_{v-1}, \frac{d}{d x}\left(x^{-v} Z_{v}\right)=-x^{-v} K_{v-1}$,

where $Z_{v}=J_{v}$ or $N_{v}$, we get

$\int_{0}^{\infty} \frac{\pi}{2} \frac{K_{m+h}(\sqrt{2 \alpha} R)}{K_{m+h}(\sqrt{2 \alpha} r)}\left(J_{m+h}(\lambda R) N_{m+h}(\lambda r)-J_{m+h}(\lambda r) N_{m+h}(\lambda R)\right) R d R=-\frac{1}{2 \alpha+\lambda^{2}}$.

The result follows immediately from the Weber's inversion transform (see [7]). This ends the proof.

Letting $k=0$ in Lemma 2.1, we have

Lemma 2.4. Let $\sigma(d y)$ be the $d-1$ dimensional volume measure on $\partial B_{r}(d \geq 2)$, then

$\int_{\partial B_{r}} C_{m}^{h}(\cos \theta) \sigma(d y)=\left\{\begin{array}{c}\frac{2 \pi^{\frac{d}{2}} r^{d-1}}{\Gamma\left(\frac{d}{2}\right)}, m=0 \\ 0, m \geq 1\end{array}\right.$

where $\theta=\angle x 0 y, \quad x \in \mathbb{R}^{d}$.

Lemma 2.5. Let $x, c \in \mathbb{R}^{d}(d \geq 2), \sigma(d y)$ be the $d-1$ dimensional volume measure on $\partial B_{r}$, then

$\int_{\partial B_{r}} e^{c \cdot y} C_{m}^{h}(\cos \theta) \sigma(d y)=2(r \pi)^{\frac{d}{2}}\left(\frac{|c|}{2}\right)^{-h} I_{m+h}(|c| r) C_{m}^{h}(\cos \angle c 0 x)$.

where $\theta=\angle x 0 y, \quad x \in \mathbb{R}^{d}$.

Proof. Using (1.5) in Yin [4] and Lemma 4 in [6, P.245].

\section{HITTING SPHERE FOR BROWNIAN MOTION}

In this section, we will give the joint density of the first hitting time and the first hitting place of a sphere by Brownian motion is derived based on the Laplace-Gegenbauer transform obtained in Wendel [1]. The result in Theorem 3.1 is due to Hsu [5], which was obtained by solving the heat equation with Dirichlet boundary condition satisfied by the transition density function of the Brownian motion in a ball.

For the interior problem we have

Theorem 3.1. For $x, y \in \mathbb{R}^{d}(d \geq 2),|x|<r,|y|=r$ and $t>0$, then

(1) for $d \geq 3$, we have

$P_{x}\left(T_{r} \in d t, X\left(T_{r}\right) \in d y\right) / d t \sigma(d y)=-\sum_{m=0}^{\infty} \sum_{n=1}^{\infty} \frac{\Gamma(h)(m+h) C_{m}^{h}(\cos \theta) q_{m, n} J_{m+h}\left(\frac{|x|}{r} q_{m, n}\right)}{2 \pi^{h+1} r^{h+3}|x|^{h} J_{m+h}^{\prime}\left(q_{m, n}\right)} e^{-\frac{q_{m, n}^{2}}{2 r^{2}} t} ;$

(2) for $d=2$, we have

$P_{x}\left(T_{r} \in d t, X\left(T_{r}\right) \in d y\right) / d t \sigma(d y)=-\sum_{n=1}^{\infty} \frac{q_{0, n} J_{0}\left(\frac{|x|}{r} q_{0, n}\right)}{2 \pi r^{3} J_{0}^{\prime}\left(q_{0, n}\right)} e^{-\frac{q_{0, n}^{2}}{2 r^{2} t}}-\sum_{m=1}^{\infty} \sum_{n=1}^{\infty} \frac{q_{m, n} \cos (m \theta) J_{m+h}\left(\frac{|x|}{r} q_{m, n}\right)}{\pi r^{3} J_{m}^{\prime}\left(q_{m, n}\right)} e^{-\frac{q_{m, n}^{2}}{2 r^{2}} t}$,

where $\theta=\angle x 0 y, \sigma$ is the $d-1$ dimensional volume measure on $\partial B_{r}$.

Proof. Let us denote by $H(t, y)$ the right hand side of (3.1). For $\alpha>0$ and integer $k \geq 0$, using Lemmas 2.1 and 2.2 we have 


$$
\begin{aligned}
& \int_{0}^{\infty} \int_{\partial B_{r}} e^{-\alpha t} C_{k}^{h}(\cos \theta) H(t, y) d t \sigma(d y)=-\frac{\Gamma(h)}{2 \pi^{h+1} r^{h+3}|x|^{h}} \sum_{m=0}^{\infty} \sum_{n=1}^{\infty} \frac{(m+h) q_{m, n} J_{m+h}\left(\frac{|x|}{r} q_{m, n}\right)}{J_{m+h}^{\prime}\left(q_{m, n}\right)} \\
& \quad \times \int_{0}^{\infty} e^{-\alpha t-\frac{q_{m, n}^{2}}{2 r^{2}} d t} d t \times \int_{\partial B_{r}} C_{k}^{h}(\cos \theta) C_{m}^{h}(\cos \theta) \sigma(d y)=-\frac{2 r^{h} C_{k}^{h}(1)}{|x|^{h}} \sum_{n=1}^{\infty} \frac{q_{k, n} J_{k+h}\left(\frac{|x|}{r} q_{k, n}\right)}{\left(2 r^{2} \alpha+q_{k, n}^{2}\right) J^{\prime}{ }_{k+h}\left(q_{k, n}\right)} \\
& =\left(\frac{r}{|x|}\right)^{h} C_{k}^{h}(1) \frac{I_{k+h}(\sqrt{2 \alpha}|x|)}{I_{k+h}(\sqrt{2 \alpha} r)} .
\end{aligned}
$$

On the other hand, from (3) in Wendel [1] we get

$$
\int_{0}^{\infty} \int_{\partial B_{r}} e^{-\alpha t} C_{k}^{h}(\cos \theta) P_{x}\left(T_{r} \in d t, X\left(T_{r}\right) \in d y\right)=\frac{r^{h} C_{k}^{h}(1) I_{k+h}(\sqrt{2 \alpha}|x|)}{|x|^{h} I_{k+h}(\sqrt{2 \alpha} r)} .
$$

It follows from (3.3) and (3.4) and the uniqueness that $P_{x}\left(T_{r} \in d t, B\left(T_{r}\right) \in d y\right)=H(t, y) d t \sigma(d y)$.

This proves (3.1). Eq. (3.2) can be proved along the same lines of (3.1) and thus the proof is omitted.

Remark 3.1. When $r=1$, the result (3.1) coincides with (13) in Hsu [5].

Corollary 3.1. For $x \in \mathbb{R}^{d}(d \geq 2), \quad|x|<r$, and $t>0$, then

$$
P_{x}\left(T_{r} \in d t\right) / d t=\sum_{n=1}^{\infty} \frac{q_{0, n}}{r^{2} J_{h+1}\left(q_{0, n}\right)}\left(\frac{|x|}{r}\right)^{-h} J_{h}\left(\frac{|x|}{r} q_{0, n}\right) e^{-\frac{q_{0, n}^{2}}{2 r^{2}} t} .
$$

Proof. Integrating (3.1) or (3.2) with respect to $y \in \partial B_{r}$, using Lemma 2.4 and $J^{\prime}{ }_{h}\left(q_{0, n}\right)=-J_{h+1}\left(q_{0, n}\right)$.

For the exterior problem we have

Theorem 3.2. For $x, y \in \mathbb{R}^{d}(d \geq 2),|x|<r,|y|=r$ and $t>0$, then

(1) for $d \geq 3$, we have

$$
\begin{aligned}
& P_{x}\left(T_{r} \in d t, X\left(T_{r}\right) \in d y, T_{r}<\infty\right) / d t \sigma(d y)=-\frac{\Gamma\left(\frac{d}{2}\right)}{2 r h \pi^{\frac{d}{2}+1}(r|x|)^{h}} \sum_{m=0}^{\infty}(m+h) C_{m}^{h}(\cos \theta) \\
& \quad \times \int_{0}^{\infty} \frac{\lambda\left(J_{m+h}(\lambda|x|) N_{m+h}(\lambda r)-J_{m+h}(\lambda r) N_{m+h}(\lambda|x|)\right)}{J^{2}(\lambda r)+N_{m+h}^{2}(\lambda r)} e^{-\frac{1}{2} \lambda^{2} t} d \lambda
\end{aligned}
$$

(1) for $d=2$, we have

$$
\begin{aligned}
& P_{x}\left(T_{r} \in d t, X\left(T_{r}\right) \in d y, T_{r}<\infty\right) / d t \sigma(d y)=-\sum_{m=0}^{\infty} \frac{|x| D(m,|x|)}{\pi r} C_{m}^{0}(\cos \theta) \\
& \quad \times \int_{0}^{\infty} \frac{\lambda\left(J_{m}(\lambda|x|) N_{m}(\lambda r)-J_{m}(\lambda r) N_{m}(\lambda|x|)\right)}{J_{m}^{2}(\lambda r)+N_{m}^{2}(\lambda r)} e^{-\frac{1}{2} \lambda^{2} t} d \lambda,
\end{aligned}
$$

Where $\theta=\angle x 0 y, \sigma$ is the $d-1$ dimensional volume measure on $\partial B_{r}$ and $D(m,|x|)=\frac{m}{2 \pi|x|}$, if $m \neq 0 ; \frac{1}{2 \pi|x|}$, if $m=0$.

Proof. Let us denote by $G(t, y)$ the right hand side of (3.6). For $\alpha>0$ and integer $k \geq 0$, using Lemmas 2.1 and 2.3 we have

$$
\begin{aligned}
\int_{0}^{\infty} & \int_{\partial B_{r}} e^{-\alpha t} C_{k}^{h}(\cos \theta) G(t, y) d t \sigma(d y)=-\frac{\Gamma\left(\frac{d}{2}\right)}{2 r h \pi^{\frac{d}{2}+1}(r|x|)^{h}} \sum_{m=0}^{\infty}(m+h) \int_{\partial B_{r}} C_{k}^{h}(\cos \theta) C_{m}^{h}(\cos \theta) \sigma(d y) \\
& \times \int_{0}^{\infty} \frac{\lambda\left(J_{m+h}(\lambda|x|) N_{m+h}(\lambda r)-J_{m+h}(\lambda r) N_{m+h}(\lambda|x|)\right)}{J^{2}(\lambda r)+N_{m+h}^{2}(\lambda r)} e^{-\frac{1}{2} \lambda^{2} t-\alpha t} d \lambda d t=\left(\frac{r}{|x|}\right)^{h} C_{k}^{h}(1) \frac{K_{k+h}(\sqrt{2 \alpha}|x|)}{K_{k+h}(\sqrt{2 \alpha} r)} .
\end{aligned}
$$

On the other hand, from (6) in Wendel [1] we get 
$\int_{0}^{\infty} \int_{\partial B_{r}} e^{-\alpha t} C_{k}^{h}(\cos \theta) P_{x}\left(T_{r} \in d t, X\left(T_{r}\right) \in d y, T_{r}<\infty\right)=\left(\frac{r}{|x|}\right)^{h} C_{k}^{h}(1) \frac{K_{k+h}(\sqrt{2 \alpha}|x|)}{K_{k+h}(\sqrt{2 \alpha} r)}$.

It follows from (3.8) and (3.9) and the uniqueness that $P_{x}\left(T_{r} \in d t, B\left(T_{r}\right) \in d y\right)=G(t, y) d t \sigma(d y)$.

This proves (3.6). (3.7) can be proved along the same lines as the case (3.6) and will be omitted.

The following corollary follows immediately from Theorem 3.2 and Lemma 2.4.

Corollary 3.2. For $x \in \mathbb{R}^{d}(d \geq 2),|x|<r$ and $t>0$, then

$P_{x}\left(T_{r} \in d t, T_{r}<\infty\right) / d t=-\frac{1}{\pi}\left(\frac{r}{|x|}\right)^{h} \int_{0}^{\infty} \frac{\lambda\left(J_{h}(\lambda|x|) N_{h}(\lambda r)-J_{h}(\lambda r) N_{h}(\lambda|x|)\right)}{J_{h}^{2}(\lambda r)+N_{h}^{2}(\lambda r)} e^{-\frac{1}{2} \lambda^{2} t} d \lambda$.

\section{HITTING SPHERE FOR BROWNIAN MOTION WITH DRIFT}

In this section, we will give the joint density of the first hitting time and the first hitting place of a sphere by Brownian motion with constant drift. The results can be proved, as in the last section, by inverting the Laplace-Gegenbauer transform for Brownian motion with drift obtained in Yin [4]. Or, using Girsanov's change of measure theorem for Brownian motion. We give the results without proof.

For the interior problem we have

Theorem 4.1. For $c, x, y \in \mathbb{R}^{d}(d \geq 2),|x|<r,|y|=r$ and $t>0$, then

(1) for $d \geq 3$, we have

$$
P_{x}^{c}\left(T_{r} \in d t, X\left(T_{r}\right) \in d y\right) / d t \sigma(d y)=-e^{c \cdot(y-x)-\frac{1}{2} c c^{2} t} \sum_{m=0}^{\infty} \sum_{n=1}^{\infty} \frac{\Gamma(h)(m+h) C_{m}^{h}(\cos \theta) q_{m, n} J_{m+h}\left(\frac{|x|}{r} q_{m, n}\right)}{2 \pi^{h+1} r^{h+3}|x|^{h} J_{m+h}^{\prime}\left(q_{m, n}\right)} e^{-\frac{q_{m, n}^{2}}{2 r^{2}} t} ;
$$

(2) for $d=2$, we have

$$
P_{x}^{c}\left(T_{r} \in d t, X\left(T_{r}\right) \in d y\right) / d t \sigma(d y)=-e^{c \cdot(y-x)-\frac{1}{2} \mid c t^{2} t}\left(\sum_{n=1}^{\infty} \frac{q_{0, n} J_{0}\left(\frac{|x|}{r} q_{0, n}\right)}{2 \pi r^{3} J_{0}^{\prime}\left(q_{0, n}\right)} e^{-\frac{q_{0, n}^{2}}{2 r^{2}}}+\sum_{m=1}^{\infty} \sum_{n=1}^{\infty} \frac{q_{m, n} \cos (m \theta) J_{m}\left(\frac{|x|}{r} q_{m, n}\right)}{\pi r^{3} J_{m}^{\prime}\left(q_{m, n}\right)} e^{-\frac{q_{m, n}^{2}}{2 r^{2}} t}\right),
$$

where $\theta=\angle x 0 y, \sigma$ is the $d-1$ dimensional volume measure on $\partial B_{r}$.

Corollary 4.1. For $c, x \in \mathbb{R}^{d}(d \geq 2), \quad|x|<r$ and $t>0$, then

(1) for $d \geq 3$, we have

$$
\begin{aligned}
& P_{x}^{c}\left(T_{r} \in d t\right) / d t=-e^{-c \cdot x-\frac{1}{2}|c|^{2} t} \frac{2^{h} \Gamma(h)}{r^{2}(|c| \cdot|x|)^{h}} \\
& \quad \times \sum_{m=0}^{\infty} \sum_{n=1}^{\infty} \frac{(m+h) C_{m}^{h}(\cos \angle c 0 x) q_{m, n} I_{m+h}(r|c|) J_{m+h}\left(\frac{|x|}{r} q_{m, n}\right)}{J^{\prime}{ }_{m+h}\left(q_{m, n}\right)} e^{-\frac{q_{m, n}^{2}}{2 r^{2}}} ;
\end{aligned}
$$

(2) for $d=2$, we have

$$
\begin{aligned}
& P_{x}^{c}\left(T_{r} \in d t\right) / d t \\
& =-e^{-c \cdot x-\frac{1}{2}|c|^{2} t} \sum_{n=1}^{\infty} \frac{q_{0, n} I_{0}(r|c|) J_{0}\left(\frac{|x|}{r} q_{0, n}\right)}{r^{2} J_{0}^{\prime}\left(q_{0, n}\right)} e^{-\frac{q_{0, n}^{2} t}{2 r^{2}}} \\
& -e^{-c \cdot x-\frac{1}{2}|c|^{2} t} \sum_{m=1}^{\infty} \sum_{n=1}^{\infty} \frac{2 q_{m, n} \cos (m \angle c 0 x) I_{m}(r|c|) J_{m}\left(\frac{|x|}{r} q_{m, n}\right)}{r^{2} J_{m}^{\prime}\left(q_{m, n}\right)} e^{-\frac{q_{m, n}^{2}}{2 r^{2}} t}
\end{aligned}
$$

Proof. Integrating (3.1) or (3.2) with respect to $y \in \sum^{d-1}(0, r)$ and using Lemma 2.5.

For the exterior problem we have 
Theorem 4.2. For $c, x, y \in \mathbb{R}^{d}(d \geq 2),|x|>r,|y|=r$ and $t>0$, then

(1) for $d \geq 3$, we have

$$
\begin{aligned}
& P_{x}^{c}\left(T_{r} \in d t, B\left(T_{r}\right) \in d y, T_{r}<\infty\right) / d t \sigma(d y)=-\frac{\Gamma\left(\frac{d}{2}\right) e^{c \cdot(y-x)-\frac{1}{2}|c|^{2} t}}{2 r h \pi^{\frac{d}{2}+1}(r|x|)^{h}} \sum_{m=0}^{\infty}(m+h) C_{m}^{h}(\cos \theta) \\
& \quad \times \int_{0}^{\infty} \frac{\lambda\left(J_{m+h}(\lambda|x|) N_{m+h}(\lambda r)-J_{m+h}(\lambda r) N_{m+h}(\lambda|x|)\right)}{J_{m+h}^{2}(\lambda r)+N_{m+h}^{2}(\lambda r)} e^{-\frac{1}{2} \lambda^{2} t} d \lambda
\end{aligned}
$$

(2) for $d=2$, we have

$$
\begin{aligned}
& P_{x}\left(T_{r} \in d t, B\left(T_{r}\right) \in d y, T_{r}<\infty\right) / d t \sigma(d y)=-e^{c \cdot(y-x)-\frac{1}{2}|c|^{2} t} \sum_{m=0}^{\infty} \frac{|x| D(m,|x|)}{\pi r} C_{m}^{0}(\cos \theta) \\
& \quad \times \int_{0}^{\infty} \frac{\lambda\left(J_{m}(\lambda|x|) N_{m}(\lambda r)-J_{m}(\lambda r) N_{m}(\lambda|x|)\right)}{J_{m}^{2}(\lambda r)+N_{m}^{2}(\lambda r)} e^{-\frac{1}{2} \lambda^{2} t} d \lambda
\end{aligned}
$$

where $\theta=\angle x 0 y, \sigma$ is the $d-1$ dimensional volume measure on $\partial B_{r}$ and $D(m,|x|)=\frac{m}{2 \pi|x|}$, if $m \neq 0 ; \frac{1}{2 \pi|x|}$, if $m=0$.

The following corollary follows immediately from Theorem 3.2 and Lemma 2.5.

Corollary 4.2. For $c, x \in \mathbb{R}^{d}(d \geq 2),|x|>r$ and $t>0$, then

(1) for $d \geq 3$, we have

$$
\begin{aligned}
& P_{x}^{c}\left(T_{r} \in d t, T_{r}<\infty\right) / d t=-e^{-c \cdot x-\frac{1}{2}|c|^{2} t} \frac{\Gamma(h) 2^{h}}{\pi(|c| \cdot|x|)^{h}} \sum_{m=0}^{\infty}(m+h) I_{m+h}(r|c|) C_{m}^{h}(\cos \angle c 0 x) \\
& \times \int_{0}^{\infty} \frac{\lambda\left(J_{m+h}(\lambda|x|) N_{m+h}(\lambda r)-J_{m+h}(\lambda r) N_{m+h}(\lambda|x|)\right)}{J_{m+h}^{2}(\lambda r)+N_{m+h}^{2}(\lambda r)} e^{-\frac{1}{2} \lambda^{2} t} d \lambda
\end{aligned}
$$

(2) for $d=2$, we have

$$
\begin{aligned}
& P_{x}^{c}\left(T_{r} \in d t, T_{r}<\infty\right) / d t=-e^{-c \bullet x-\frac{1}{2}|c|^{2} t}\left(\frac{1}{\pi} I_{0}(r|c|)+\sum_{m=1}^{\infty} \frac{m}{\pi} I_{m}(r|c|) C_{m}^{0}(\cos \angle c 0 x)\right) \\
& \times \int_{0}^{\infty} \frac{\lambda\left(J_{m}(\lambda|x|) N_{m}(\lambda r)-J_{m}(\lambda r) N_{m}(\lambda|x|)\right)}{J_{m}^{2}(\lambda r)+N_{m}^{2}(\lambda r)} e^{-\frac{1}{2} \lambda^{2} t} d \lambda .
\end{aligned}
$$

\section{ACKNOWLEDGEMENTS}

The authors would like to thank the anonymous referees for their helpful comments and suggestions to improve the earlier version of the paper. This research was supported by the National Natural Science Foundation of China (No. 10771119) and the Key Project of Chinese Ministry of Education (No. 206091).

\section{REFERENCES}

[1] J. G. Wendel, "Hitting spheres with Brownian motion", Ann. Probab. vol. 8, pp. 164-169, 1980.
[2] C. Betz and H. Gzyl, "Hitting spheres from the exterior", Ann. Probab., vol. 22, pp. 177-179, 1994.

[3] C. Betz and H. Gzyl, "Hitting spheres with Brownian motion and Sommerfeld's radiation condition", J. Math. Anal. Appl. vol. 182, pp. 301-308, 1994.

[4] C. C. Yin, "The joint distribution of the hitting time and place to a sphere or spherical shell for Brownian motion with drift", Stat. Probab. Lett. vol. 42, pp. 367-373, 1999.

[5] P. Hsu, "Brownian exit distributions of a ball, Seminar on stochastic processes", Boston: Birkhauser, 1986.

[6] A. Erdelyi, W. Magnus, F. Oberhettinger and F. G. Tricomi, Higher Transcenden -tal Functions, New York: McGraw-Hill, vol. 2, 1953.

[7] B. Davies, Integral Transforms and Their Applications, $2^{\text {nd }}$ ed. New York: Springer -Verlag, 1985. 